\title{
Neutralización del efecto hemorrágico inducido por veneno de Bothrops asper (Serpentes: Viperidae) por extractos de plantas tropicales
}

\author{
Oscar Castro ${ }^{1}$, José María Gutiérrez ${ }^{2}$, Mariano Barrios ${ }^{1}$, Ian Castro ${ }^{3}$, Marjorie Romero² y \\ Eduardo Umaña ${ }^{1}$ \\ 1 Departamento de Química, Universidad Nacional, Heredia, Costa Rica. \\ 2 Instituto Clodomiro Picado, Facultad de Microbiología, Universidad de Costa Rica, 2060 San José, Costa Rica; Fax \\ 506-2920485, dirección electrónica: jgutierr@cariari.ucr.ac.cr. \\ 3 Laboratorio de Productos Naturales, Universidad de San Carlos, Sao Paulo, Brasil
}

Recibido 18-VIII-1998 Corregido 9-III-1999 Aceptado 18-III-1999

\begin{abstract}
Organic extracts representing 48 species included in 30 families of Costa Rican tropical plants were evaluated for their ability to neutralize hemorrhagic activity induced by the venom of the snake Bothrops asper. A bioassay in mice was used, based on intradermal injection of either venom or venom-extract mixtures followed by the measurement of hemorrhagic areas. Total inhibition of hemorrhage was observed with the ethanolic, ethyl acetate and aqueous extracts of Bursera simaruba, Clusia torresii, C. palmana, Croton draco, Persea americana. Phoebe brenesii, Pimenta dioica, Sapindus saponaria, Smilax cuculmeca and Virola koschnyi. Chemical analysis of these extracts identified catequines, flavones, anthocyanines and condensated tannins, which may be responsible for the inhibitory effect observed, probably owing to the chelation of the zinc required for the catalytic activity of venom's hemorrhagic metalloproteinases.
\end{abstract}

Key words: Anti-hemorrhagic plants, anti-venom plants, snake venom, Bothrops asper, flavonoids, tannins.

Los venenos de serpientes de la familia Viperidae inducen un complejo cuadro de alteraciones fisiopatológicas que incluyen tanto efectos locaies, en el sitio de la mordedura, como sistémicos (Rosenfeld 1971). La serpiente Bothrops asper, conocida popularmente como terciopelo, barba amarilla o nauyaca, es la que ocasiona el mayor número de accidentes en Centroamérica (Bolaños 1982, Campbell y Lamar 1989, Gutiérrez 1995). Su veneno provoca importantes efectos locales (hemorragia, edema y necrosis), así como diversas alteraciones sistémicas (coagulopatías, hemorragia, choque cardiovascular e insuficiencia renal aguda) (Gutiérrez 1995).

El único tratamiento científicamente validado para los envenenamientos ofídicos es la administración parenteral de antivenenos producidos en caballos o en ovejas (Warrell 1996). Los antivenenos son altamente eficaces en la neutralización de los efectos sistémicos inducidos por los venenos. Sin embargo, la neutralización de los efectos locales se logra sólo parcialmente, debido a la rapidez con que estos efectos se desencadenan y, en muchos casos, al retardo en la administración del antiveneno (Gutiérrez y Lomonte 1989, Gutiérrez et al. 1998). Por ello, se ha planteado la necesidad de buscar sustancias naturales o sintéticas que neutralicen las toxinas responsables de estos efectos locales y que puedan utilizarse como complemento de la seroterapia. Los extractos de plantas tropicales constituyen importantes reservorios de potenciales sustancias inhibidoras de toxinas de venenos y una reciente revisión señala que se ha 
descrito algún tipo de actividad anti-veneno en 578 especies de plantas superiores (Martz 1992).

La hemorragia local y sistémica constituye un efecto central en la fisiopatología del envenenamiento por $B$. asper (Gutiérrez y Lomonte 1989, Gutiérrez 1995). Este efecto es mediado por una serrie de metaloproteinasas dependientes de zinc (Borkow et al. 1993, Gutiérrez et al. 1995, Franceschi et al. 1999), las cuales degradan componentes de la lámina basal de vasos capilares, ocurriendo como consecuencia la extravasación (Moreira et al. 1992, 1994). Dada la relevancia de este efecto, y dado que se ha demostrado actividad anti-hemorrágica en extractos de algunas plantas sudamericanas (Ferreira et al. 1992, Melo et al. 1994), en el presente trabajo se evaluó la capacidad de extractos de 48 especies de plantas para neutralizar la actividad hemorrágica del veneno crudo de B. asper. Además, se identificaron estructuras químicas en estos extractos, principalmente de naturaleza flavonoide, como posibles responzables del efecto inhibitorio observado.

\section{MATERIALES Y MÉTODOS}

Veneno: Se utilizó el veneno obtenido de más de 50 especímenes adultos de $B$. asper recolectados en la región Pacífica de Costa Rica y mantenidos en el serpentario del Instituto Clodomiro Picado. Una vez obtenido, el veneno se liofilizó y se guardó a $-40{ }^{\circ} \mathrm{C}$ hasta su utilización.

Plantas: Tomando como base criterios etnobotánicos y sistemáticos, se seleccionaron las siguientes 48 especies de plantas, distribuídas en 30 familias: Asteraceae (Bidens pilosa, Mikania guaco, Neurolaena lobata, Vernonia patens), Asclepiadaceae (Asclepias curassavica), Annonaceae (Annona purpurea, Rollinia mucosa), Apocinaceae (Allamanda cathartica), Aristolochiaceae (Aristolochia grandiflo$r a$ ), Burseraceae (Bursera simaruba), Bignoniaceae (Tabebuia palmeri), Clusiaceae (Clusia palmana, Clusia torresii, Hypericum strictum, Hypericum irazuensis,Tovomita sp.), Cucurbitaceae (Fevillea cordifolia), Ca- prifoliaceae (Viburnum costaricanum), Combretaceae (Terminalia oblonga), Commeliniaceae (Zebrina pendula), Euphorbiaceae (Croton draco), Flacourtiaceae (Casearia silvestris), Lauraceae (Ocotea holdridgeina, $O$. valerianoides, $O$. insularis, Phoebe brenesii, Persea americana var Hass), Lamiaceae (Satureja brownei), Leguminoseae (Platymiscium pleiostachyum, Senna emarginata, Gliricidia sepium), Liliaceae (Sansevieria guineensis), Loganiaceae (Buddleia nitida), Meliaceae (Cedrela tonduzii), Moraceae (Dorstenia contrajerba), Myristicaceae (Virola koschnyi), Myrtaceae (Pimenta dioica), Piperaceae (Pimenta darienensis), Phytolacaceae (Petiveria alliaceae), Rubiaceae (Genipa americana), Simaroubaceae (Picramnia antidesma, $P$. teapensis), Sapindaceae (Sapindus saponaria), Smilacaceae (Smilax cuculmeca) y Verbenaceae (Citharexylum macrodenium, Duranta repens, Stachytarpheta jamaicensis, Verbena litoralis) (Cuadro 1). Estas plantas fueron recolectadas y clasificadas por Luis J. Poveda y Oscar Castro mediante comparación directa con muestras depositadas en el Herbario del Museo Nacional de Costa Rica. Inicialmente se recolectaron muestras cuyo peso fue de aproximadamente un kilogramo. Posteriormente, de las plantas que presentaron actividad neutralizante de la hemorragia, se recolectaron hasta $3 \mathrm{Kg}$.

Elaboración de extractos: Se efectuaron extracciones durante $48 \mathrm{hr}$ por maceración en frío con una mezcla de etanol: agua $(85: 15)$. Este procedimiento se repitió dos veces y los extractos alcohólicos acuosos obtenidos se concentraron hasta consistencia siruposa mediante destilación al vacío y a temperaturas inferiores a $45^{\circ} \mathrm{C}$. Para los ensayos biológicos se entregaron extractos liofilizados en muestras que pesaron entre $1 \mathrm{y} 2 \mathrm{~g}$. Los extractos que mostraron actividad inhibitoria de la hemorragia fueron fraccionados mediante particiones sucesivas con los siguientes solventes de polaridad creciente: hexano (A), diclorometano (B) y acetato de etilo (C). Todos estos extractos, incluyendo los 
remanentes alcohólicos acuosos (D), fueron evaluados para ubicar dónde se encontraba la actividad anti-hemorrágica.

Aislamiento e identificación de compuestos: Se emplearon fundamentalmente técnicas de separación y purificación cromatográfica en columna y en placa preparativa, empleando como soporte gel de sílice. Los compuestos fueron caracterizados mediante el análisis de los correspondientes datos espectroscópicos de: ultravioleta (UV), infrarrojo (IR), resonancia magnética nuclear de protio ( $\left.{ }^{1} \mathrm{H} \mathrm{RMN}\right)$, resonancia magnética nuclear de carbono-13 ( $\left.{ }^{13} \mathrm{C} \mathrm{RMN}\right)$, incluyendo en algunos casos Análisis Bidimensional de Resonancia Homonuclear ( $\left.{ }^{1} \mathrm{H}-\mathrm{H}-\mathrm{RMN}\right)$ y Heteronuclear $\left(\mathrm{H}-{ }^{13} \mathrm{C}\right.$ RMN $)$. Para las ${ }^{1} \mathrm{H}$ RMN y ${ }^{13} \mathrm{C}$ RMN se contó con instrumental espectroscópico de alta resolución del Laboratorio de Investigación en Productos Naturales de la Universidad de San Carlos, Sao Paulo, Brasil.

Ensayo biológico: Para la determinación de actividad hemorrágica, y su correspondiente neutralización, se utilizó la técnica en piel descrita por Kondo et al. (1960), de acuerdo a las modificaciones de Gutiérrez et al. (1985). Inicialmente se determinó la actividad hemorrágica del veneno de $B$. asper, mediante inyecciones de dosis crecientes de veneno, disueltas en $0.1 \mathrm{~mL}$ de $0.14 \mathrm{M} \mathrm{NaCl}$, $0.04 \mathrm{M}$ fosfatos, pH 7.2 (SAF). Estas inyecciones se efectuaron por la vía intradérmica, en la región ventral de ratones Swiss-Webster de 18 a $22 \mathrm{~g}$. Los ratones control recibieron $0.1 \mathrm{~mL}$ de SAF. Dos horas después de las inyecciones los ratones se sacrificaron mediante inhalación de éter, sus pieles fueron removidas y el área hemorrágica en la región interna de la piel fue medida. La Dosis Hemorrágica Mímina (DHM) corresponde a la cantidad de veneno que induce un área hemorrágica de $10 \mathrm{~mm}$ de diámetro.

Para los estudios de neutralización se empleó una dosis de reto de veneno correspondiente a 10 DHM. Una cantidad constante de veneno se incubó con una cantidad de extracto a una razón veneno: extracto de 1:50 (peso: peso), manteniendo un volumen total constante. Se prepararon controles que incluyeron veneno sin extracto, extracto sin veneno y SAF. Las mezclas se incubaron a temperatura ambiente $\left(20-23{ }^{\circ} \mathrm{C}\right)$ durante $30 \mathrm{~min}$ y de cada mezcla se inyectaron grupos de tres ratones con $0.1 \mathrm{~mL}$, conteniendo una dosis de reto de veneno. Dos horas después de la inyección, los ratones se sacrificaron y el área de la lesión hemorrágica en piel se cuantificó de la manera descrita. La actividad anti-hemorrágica se expresó en términos porcentuales, donde el $100 \%$ corresponde a una neutralización total del efecto y el $0 \%$ corresponde a un área hemorrágica cuyo diámetro es igual al de la lesión inducida por el veneno solo. En los experimentos de neutralización también se evaluó la toxicidad de los extractos, en términos de letalidad o desarrollo de algún tipo de lesión en piel.

\section{RESULTADOS}

Actividad anti-hemorrágica: El veneno de $B$. asper presentó una Dosis Hemorrágica Mínima (DHM) de $2 \mu \mathrm{g}$, por lo que en experimentos de neutralización se empleó una dosis de reto de veneno de $20 \mu \mathrm{g}$, los cuales se incubaron con $1 \mathrm{mg}$ de los extractos, para mantener una razón por peso de 1:50. Ninguno de los extractos evaluados causó la muerte de los ratones inyectados ni indujo lesiones locales en piel en las condiciones experimentales utilizadas en este estudio. De los 52 extractos hidroalcohólicos evaluados, 10 de ellos neutralizaron en su totalidad la actividad hemorrágica del veneno, en tanto otros 8 indujeron una neutralización parcial (Cuadro 1).

Para identificar la polaridad del extracto donde se concentraba la mayor actividad, los extractos que neutralizaron el veneno se suspendieron en una mezcla etanol/agua (1:1) y luego se extrajeron mediante particiones sucesivas con los siguientes solventes de polaridad creciente: hexano, diclorometano y acetato de etilo. En general se observó que la actividad 


\section{CUADRO 1}

Evaluación de la capacidad neutralizante de extractos crudos hidroalcohólicos derivados de plantas hacia el efecto hemorrágico inducido por el veneno de Bothrops asper.

\begin{tabular}{|c|c|c|c|c|c|}
\hline Planta & Parte* & Neutralización ** & Planta & Parte* & Neutralización $* *$ \\
\hline Allamanda catartica & $\mathrm{H}$ & - & Ocotea valerianoides & $\mathrm{P}$ & - \\
\hline Annona purpurea & M & - & Persea americana & $\mathrm{S}$ & ++ \\
\hline Aristolochia grandiflora & $\mathrm{H}$ & - & Petiveria alliaceae & $\mathrm{H}, \mathrm{T}$ & + \\
\hline Asclepias curassavica & $\mathrm{H}$ & - & Petiveria alliaceae & $\mathrm{R}$ & - \\
\hline Bidens pilosa & $\mathrm{H}$ & - & Phoebe brenesii & $\mathrm{C}$ & ++ \\
\hline Buddleia nitida & $\mathrm{H}$ & - & Picramnia antidesma & $\mathrm{H}, \mathrm{C}$ & + \\
\hline Bursera simaruba & $\mathrm{C}$ & ++ & Picramnia teapensis & $\mathrm{H}, \mathrm{C}, \mathrm{F}$ & + \\
\hline Casearia silvestris & $\mathrm{H}, \mathrm{C}$ & - & Pimenta dioica & $\mathrm{H}$ & ++ \\
\hline Cedrela tonduzii & $\mathrm{H}, \mathrm{C}$ & - & Platymiscium pleiostachyum & $\mathrm{H}, \mathrm{M}$ & - \\
\hline Citharexylum macrodenium & $\mathrm{H}$ & - & Rollinia mисоsa & $\mathrm{H}, \mathrm{M}$ & - \\
\hline Clusia torresii & $\mathrm{H}, \mathrm{F}$ & ++ & Sanseveria guineensis & $\mathrm{H}$ & - \\
\hline Clusia palmana & $\mathrm{H}, \mathrm{Bf}$ & ++ & Sapindus saponaria & $\mathrm{H}$ & ++ \\
\hline Croton draco & $\mathrm{C}, \mathrm{L}$ & ++ & Satureja brownei & $\mathrm{H}$ & + \\
\hline Dorstenia contrajerba & $\mathrm{H}, \mathrm{R}$ & - & Senna emarginata. & $\mathrm{S}$ & - \\
\hline Duranta repens & $\mathrm{H}$ & - & Smilax cuculmeca & $\mathrm{R}$ & ++ \\
\hline Duranta repens & $\mathrm{F}$ & + & Stachytarpheta jamaicensis & $\mathrm{H}$ & + \\
\hline Fevillea cordifolia & $\mathrm{S}$ & - & Tabebuia palmeri & $\mathrm{C}, \mathrm{M}$ & - \\
\hline Genipa americana & $\mathrm{H}, \mathrm{P}$ & - & Terminalia oblonga & $\mathrm{H}$ & - \\
\hline Gliricidia sepium & $\mathrm{H}$ & - & Tovomita $s p$ & $\mathrm{Bf}$ & - \\
\hline Hypericum irazuensis & $\mathrm{H}, \mathrm{C}$ & + & Verbena littoralis & $\mathrm{H}$ & - \\
\hline Hypericum strictum & $\mathrm{H}, \mathrm{C}$ & - & Vernonia patens & $\mathrm{H}$ & - \\
\hline Mikania guaco & $\mathrm{H}, \mathrm{T}$ & - & Viburnum costaricanum & $\mathrm{H}$ & - \\
\hline Neurolaena lobata & $\mathrm{H}$ & - & Virola koschnyi & $\mathrm{C}$ & ++ \\
\hline Ocotea holdridgenia & $\mathrm{P}$ & - & Virola koschnyi & $\mathrm{P}$ & - \\
\hline Ocotea insularis & $\mathrm{H}$ & - & Zebrina pendula & $\mathrm{H}$ & + \\
\hline
\end{tabular}

*H: hojas; M: madera; Bf: botones florales; F: flores; C: corteza; H: hojas; L: látex; P: pulpa; R: raíz; S: semilla.

**(-) ausencia de neutralización; (+) neutralización parcial (más del 50\%, pero menos del 100\%); (++) neutralización total (100\%).

anti-hemorrágica se concentró principalmente en las fracciones de acetato de etilo y en los remanentes alcohólico-acuosos (Cuadro 2). Como excepción se observó que ninguna de las fracciones derivadas de Sapindus saponaria inhibió totalmente esta actividad (Cuadro 2).

Caracterización química de los extractos: Los extractos de acetato de etilo de Phoebe brenesii (corteza), Clusia palmana (hojas), C. torresii (hojas), Bursera simaruba (corteza), Croton draco (corteza), Persea americana var Hass (semilla) y Pimenta dioica (hojas) se fraccionaron en cromatografía de columna empleando como fase estacionaria sílica gel y como eluyentes diclorometano, diclorometano/acetona (1:1), acetona y metanol. Se observó, como característica general, que las fracciones de diclorometano/acetona obtenidas de estos extractos revelaban manchas con colores típicos de compuestos polifenólicos con el reactivo de vainillina y cloruro de hierro III. Estos compuestos fueron aịslados utilizando cromatografía preparativa en sílica gel, eluyendo dos veces consecutivas con mezclas de cloroformo/metanol (90:10; 80:20). Mediante los correspondientes espectros de ${ }^{1} \mathrm{H}$ RMN y. ${ }^{13} \mathrm{C}$ RMN se identificaron flavonoides de amplia distribución en plantas, tales como quercetina (compuesto 1 de la Fig 1) en Phoebe brenesii y Pimenta dioica, vitexina (compuesto 2) y epicatequina (compuesto 3) en Clusia 


\section{CUADRO 2}

Distribución de la capacidad neutralizante hacia el efecto hemorrágico inducido por el veneno de Bothrops asper en los extractos obtenidos mediante solventes de polaridad creciente: Hexano (A), diclorometano $(B)$, acetato de etilo $(C)$ y acuoso $(D)$. Estos extractos fueron obtenidos a partir de extractos crudos hidroalcohólicos derivados de plantas que neutralizaron totalmente el efecto hemorrágico.

\begin{tabular}{|c|c|c|c|c|c|}
\hline \multirow[b]{2}{*}{ Planta } & \multirow[b]{2}{*}{ Parte } & \multicolumn{4}{|c|}{ Neutralización (\%)* } \\
\hline & & A & B & $\mathrm{C}$ & $\mathrm{D}$ \\
\hline Bursera simaruba & Corteza & 0 & 0 & 100 & 31 \\
\hline Clusia palmana & Hojas & 27 & 33 & 100 & 100 \\
\hline Clusia palmana & Frutos & 2 & 65 & 83 & 100 \\
\hline Clusia torresii & Hojas & 0 & 13 & 100 & 100 \\
\hline Clusia torresii & Frutos & 4 & 42 & 100 & 100 \\
\hline Croton draco & Corteza & 37 & 0 & 100 & 100 \\
\hline Croton draco & Látex & - & - & - & 100 \\
\hline Persea americana & Semilla & 45 & 8 & 100 & 100 \\
\hline Phoebe brenesii & Corteza & 2 & 2 & 100 & 100 \\
\hline Pimenta dioica & Hojas & 4 & 19 & 100 & 100 \\
\hline Sapindus saponaria & Hojas & 18 & 33 & 32 & 44 \\
\hline Smilax cuculmeca & Rizoma & - & - & 100 & 100 \\
\hline Virola koschnyi & Corteza & 64 & 60 & 100 & 100 \\
\hline
\end{tabular}

* La neutralización se expresa porcentualmente, correspondiendo $100 \%$ a una neutralización total de la actividad hemorrágica y $0 \%$ a una ausencia de neutralización. (-) significa que no se evaluó la capacidad neutralizante del extracto correspondiente.

torresii y $C$. palmana y catequina (compuesto 4) en Pimenta dioica y Croton draco.

De la corteza de Croton draco se aislaron también dos derivados inusuales de la catequina: el compuesto 5, previamente aislado de Croton draconoides (Aquino et al. 1991) y una catequina sustituída por una ramnosa en el carbono tres, a la cual se le ha propuesto la estructura 6. Ambos compuestos fueron justificados con base en los siguientes datos espectroscópicos y usando como modelo de comparación la catequina:

Compuesto 5: ${ }^{1} \mathrm{H}$ RMN (300 MHz, DMSO-d 6 , $\delta$ ) 8.26, 8.09, 7.92 y 7.40 (s, OH fenólicos), 6.58 (s, H-4), 6.46 (s, $\mathrm{H}-2,6), 6.06$ (d, J = 2.2Hz, H-6), 5.88 (d, J = 2.2Hz, H-8), 4.52 (d; J = 7.2Hz, H-2), 4.20 (s, br, H-3), 2.85 (dd, J = $16.3,5.2 \mathrm{~Hz}, \mathrm{H}-4), 2.53(\mathrm{dd}, \mathrm{J}=16.2,7.36 \mathrm{~Hz}, \mathrm{H}-4 *) .{ }^{13} \mathrm{C}$ RMN: 79.8 (C-2), 67.4 (C-3), 29.6 (C-4), 98.9 (C-4a), 158.1 (C-5), 96.6 (C-6), 158.0 (C-7), 95.8 (C-8), 157.2 (C8a), 135.1 (C-1'), 116.3 (C-2'), 156.2 (C-3', 5'), 105.3 (C4'), 112.4 (C-6').

Compuesto 6: ' $\mathrm{H}$ RMN (400MHz, $\left.\mathrm{CD}_{3} \mathrm{OD}, \delta\right) 6.83$ (d 1.6 $\mathrm{Hz}, \mathrm{H}-2), 6.76$ (d, J = 8.1 Hz, H-5), $6.71(\mathrm{dd} \mathrm{J}=8.1,1.7 \mathrm{~Hz}$, $\mathrm{H}-6), 5.92(\mathrm{~d}, \mathrm{~J}=2.2 \mathrm{~Hz}, \mathrm{H}-6), 5.85(\mathrm{~d}, \mathrm{~J}=2.2 \mathrm{~Hz}, \mathrm{H}-8)$, $4.56(\mathrm{~d}, \mathrm{~J}=7.5 \mathrm{~Hz}, \mathrm{H}-2), 3.97(\mathrm{dd}, \mathrm{H}-3), 3.80-3.20(5 \mathrm{H}$ ramnosa), 2.84 (dd, J =16.1, 5.4Hz, H-4), 2.50 (dd, J = $\left.16.1,8.16 \mathrm{~Hz}, \mathrm{H}-4^{*}\right), 1.12\left(\mathrm{~d}, \mathrm{~J}=6.4 \mathrm{~Hz}, \mathrm{CH}_{3}\right) .{ }^{13} \mathrm{C} \mathrm{RMN}$ : 82.9 (C-2), 69.2 (C-3), 28.6 (C-4), 100.8 (C-4a), 157.6 (C-5), 95.3 (C-6), 157.9 (C-7), 95.5 (C-8), 156.9 (C-8a), 132.2 (C-1'), 115.2 (C-2'), 146.3 (C-3',4'), 116.1 (C-5'), 120.0 (C-6'). Ramnosa: 98.0 (C-1"), 71.9 (C-2”), 71.3 (C-3"), 73.5 (C-4"), 60.6 (C-5"), 19.5 (C-6").

En este caso, los sensibles desplazamientos a campo bajo observados en ${ }^{13} \mathrm{C}$ RMN pa-

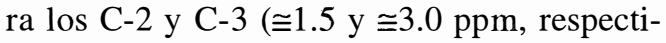
vamente), en comparación con los atribuídos a estos mismos carbonos en la catequina, sugieren la posición de la ramnosa en el carbono 3 (Markham y Ternai 1976).

Mediante cromatografía analítica comparativa se observó que los correspondientes extractos acuosos derivados de todas estas plantas concentraban también estos mismos flavonoides. Por otra parte, todos ellos, pero particularmente los derivados de corteza de Bursera simaruba, Croton draco, Phoebe brenesii y Virola koschnyi, así como los de semillas de Persea americana, se caracterizaron por ser muy ricos en taninos condensa- 
dos. Se ha descrito que la corteza de Croton draco constituye una de las fuentes más ricas de taninos naturales en Costa Rica (Céspedes et al. 1992). Con relación a Sapindus saponaria, se encontró que los extractos de diclorometano y de acetato de etilo, cuando fueron analizados mediante cromatografía de capa fina en gel de sílice mediante elución con $\mathrm{CH}_{2} \mathrm{Cl}_{2}$ y mezclas de $\mathrm{CH}_{2} \mathrm{Cl}_{2}$ /metanol (95:5; 90:10), revelaron con el reactivo de vainillina tres manchas principales con colores que sugieren la presencia de flavonoides poco polares, cuyas estructuras (compuestos $8,9 \mathrm{y}$ 10) fueron identificadas previamente en esta misma especie.

Del extracto acuoso de la semilla de aguacate (Persea americana) se identificó un singular monosacárido de siete átomos de carbono, conocido como perseína (compuesto 16), de valor quimiosistemático por ser característico de Persea. Este extracto fue también particularmente rico en taninos condensados, detectándose en algunas fracciones compuestos cuyas señales en el espectro de ${ }^{1} \mathrm{H}$ RMN y ${ }^{13} \mathrm{C}$ RMN concuerdan con proantocianidinas triméricas tales como el compuesto 17, previamente aislado de las semillas de Persea gratissima (Jacques y Haslam 1974).

En el caso de Smilax cuculmeca, y considerando que especies de este género tales como $S$. zarzaparilla son una rica fuente de saponinas esteroidales, se realizaron estudios de hidrólisis ácidas usando como patrón de comparación $S$. zarzaparilla. Estas reacciones se hicieron utilizando $\mathrm{HCl} 2 \mathrm{M}$ y sometiendo las mezclas a reflujo durante 6 horas. Ambos productos de reacción, una vez fríos, se neutralizaron con $\mathrm{Na}_{2} \mathrm{CO}_{3}$ y se extrajeron cinco veces con diclorometano, para ser posteriormente analizados por cromatografía de capa fina en gel de sílice, eluyendo con diclorometano/metanol (95:5) y revelando con ácido ortofosfórico. Los correspondientes a Smilax zarzaparilla revelaron una clara mancha amarilla ( $\operatorname{Rf} 0.40$ ), típica de una estructura esteroidal libre de azúcares (sapogenina), mientras que los de S. cucul- meca mostraron, bajo estas mismas condiciones, ausencia de productos de hidrólisis de naturaleza esteroidal. Para confirmar la ausencia de saponinas en $S$. cuculmeca se realizó la prueba de producción de espuma en agua de los extractos originales de ambas especies, obteniéndose un resultado positivo para el de $S$. zarzaparilla y negativa para $S$. cuculmeca. En concordancia con las observaciones anteriores, el análisis por espectroscopía infrarroja de cada una de estas mezclas de reacción mostró, para los productos derivados de $S$. zarzaparilla, las cuatro absorciones características de una sapogenina: $800,900,922$ y $987 \mathrm{~cm}^{-1}$, en tanto en los de $S$. cuculmeca no se observó la banda que debe aparecer cerca de $900 \mathrm{~cm}^{-1}$.

Dado que la mayoría de los compuestos de $S$. cuculmeca se concentraban en los extractos crudos sin hidrolizar C y D, se efectuaron análisis de los mismos por cromatografía de capa fina en sílica gel, utilizando una mezcla de acetato de etilo/metanol/agua (27:2.1:3.4). La presencia de manchas amarillas visibles en lámpara ultravioleta $(254 \mathrm{~nm})$, que viraban a rojo al ser expuestas a vapores de amoníaco, indicaron la presencia de al menos cuatro flavonoides de tipo antocianídico, justificados en el ultravioleta por las bandas de absorción (nm): (a) 510, 415, 340, 278; (b) 510, 415, 330, 278; (c) 490, 330, 375; y (d) 495, 480, 320, 280. Además, todos estos compuestos mostraron en el ultravioleta los desplazamientos betacrómicos, en presencia de cloruro de aluminio, típicos de antocianinas con el anillo aromático B di-hidroxilado o trihidroxilado, tales como los que caracterizan a las antocianinas cianidina y delfinidina. Considerando las evidencias anteriores y el color rojo vino que caracteriza al extracto de $S$. $\mathrm{cu}$ culmeca, en estos extractos deben predominar antocianinas comunes como la delfinidina (compuesto 13) y derivados mono y diglicosilados (compuestos 14 y 15). La distribución de todos los compuestos aislados de las plantas investigadas se resume en el Cuadro 3 y las correspondientes estructuras químicas se representan en la Fig 1. 


\section{CUADRO 3}

Metabolitos secundarios identificados en extractos de plantas que neutralizaron totalmente el efecto hemorrágico del veneno de Bothrops asper

\begin{tabular}{|c|c|c|c|}
\hline Planta & Parte & Extracto activo & Compuestos identificados \\
\hline Phoebe brenesii & Corteza & $\begin{array}{l}\text { Acetato de etilo } \\
\text { Acuoso }\end{array}$ & $\begin{array}{l}\text { Flavonoides } 1,3^{*} \\
\text { Taninos condensados }\end{array}$ \\
\hline Clusia palmana & Hojas & $\begin{array}{l}\text { Acetato de etilo } \\
\text { Acuoso }\end{array}$ & $\begin{array}{l}\text { Flavonoides 2, } 3 \\
\text { Taninos }\end{array}$ \\
\hline Clusia torresii & Hojas & Acetato de etilo & $\begin{array}{l}\text { Flavonoides 2, } 3 \\
\text { Taninos }\end{array}$ \\
\hline Pimenta dioica & Hojas & $\begin{array}{l}\text { Acetato de etilo } \\
\text { Acuoso }\end{array}$ & $\begin{array}{l}\text { Flavonoides } 1,4 \\
\text { Taninos condensados }\end{array}$ \\
\hline Bursera simaruba & Corteza & $\begin{array}{l}\text { Acetato de etilo } \\
\text { Acuoso }\end{array}$ & $\begin{array}{l}\text { Flavonoide } 3 \\
\text { Taninos condensados }\end{array}$ \\
\hline Croton draco & Corteza & $\begin{array}{l}\text { Acetato de etilo } \\
\text { Acuoso }\end{array}$ & $\begin{array}{l}\text { Flavonoides } 3,5,6 \\
\text { Taninos condensados }\end{array}$ \\
\hline Sapindus saponaria & Hojas & Diclorometano & Flavonoides $8,9,10$ \\
\hline Persea americana & Semilla & $\begin{array}{l}\text { Acetato de etilo } \\
\text { Acuoso }\end{array}$ & $\begin{array}{l}\text { Flavonoide } 3 \\
\text { Monosacárido } 16 \\
\text { Protocianidina } 17 \\
\text { Taninos condensados }\end{array}$ \\
\hline Smilax cuculmeca & Rizoma & $\begin{array}{l}\text { Acetato de etilo } \\
\text { Acuoso }\end{array}$ & $\begin{array}{l}\text { Antocianinas } \\
\text { Antocianinas } 13,14,15\end{array}$ \\
\hline Virola koschnyi & Corteza & Acuoso & Taninos condensados \\
\hline
\end{tabular}

\footnotetext{
* Los números presentados después de cada compuesto corresponden a las estructuras químicas mostradas en la Fig 1.
}

\section{DISCUSIÓN}

La capacidad de extractos de plantas de inhibir algunas actividades de venenos de serpientes ha sido ampliamente documentada (Martz 1992), aunque en muchos casos es necesario corroborar estas observaciones mediante experimentos bien controlados. Por ejemplo, extractos acuosos de Eclipta prostrata (Asteraceae) neutralizan parcialmente el efecto hemorrágico del veneno de Bothrops jararaca (Melo et al. 1994). Ferreira et al. (1992) mostraron que la ar-turmerona, aislada de extractos de raíces de Curcuma longa (Zingiberaceae), inhibió la actividad hemorrágica del mismo veneno.
Las plantas con sustancias inhibitorias de la actividad hemorrágica de venenos podrían tener aplicaciones en trastornos causados por aumento en la actividad de metaloproteinasas de matriz (MMPs), un grupo de enzimas endógenas que degradan la matriz extracelular y que son sintetizadas en diversos procesos patológicos por varios tipos de células (Woessner 1998).

En el presente estudio se utilizó un procedimiento sencillo para el estudio de la actividad hemorrágica del veneno de $B$. asper, basado en inyecciones intradérmicas de veneno, o de mezclas de veneno y extractos de plantas, evaluándose el efecto hemorrágico mediante observación macroscópica y medición de la lesión. Esta 
<smiles>O=C(C(O)=Cc1ccc(O)c(O)c1)c1c(O)cc(O)c(C2OC(CO)C(O)C(O)C(O)C2O)c1OC1C(O)C(O)C(O)C1O</smiles>

1<smiles>Oc1cc(O)c2c(c1)O[C@H](c1ccc(O)c(O)c1)[C@H](O)C2</smiles>

$\mathrm{OH}$<smiles>O=C1Cc2c(O)cc(O)cc2OC1=C=C1C=C(O)C(O)=C(O)C1</smiles>

7<smiles>COc1ccc(-c2cc(=O)c3ccccc3o2)cc1</smiles>

10<smiles></smiles>

5<smiles>CCOc1cc(-c2oc3cc(O)cc(O)c3c(=O)c2C)ccc1O</smiles>

11: $\mathrm{R}=\mathrm{CH}_{2} \mathrm{CH}_{2} \mathrm{OH}$<smiles>Cc1cc(O)cc(O)c1CC(=O)C=Cc1ccc(O)c(O)c1</smiles>

3

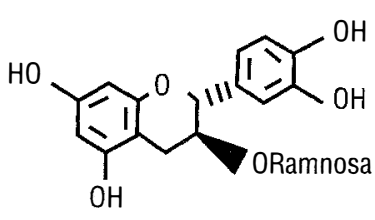

6<smiles>O=C(/C=C/c1ccc(O)c(O)c1)c1c(O)cc(O)cc1O</smiles><smiles>Cc1ccc(-c2oc3cc(OCc4ccccc4)cc(OCc4ccccc4)c3c(=O)c2OCc2ccccc2)cc1OCc1ccccc1</smiles>

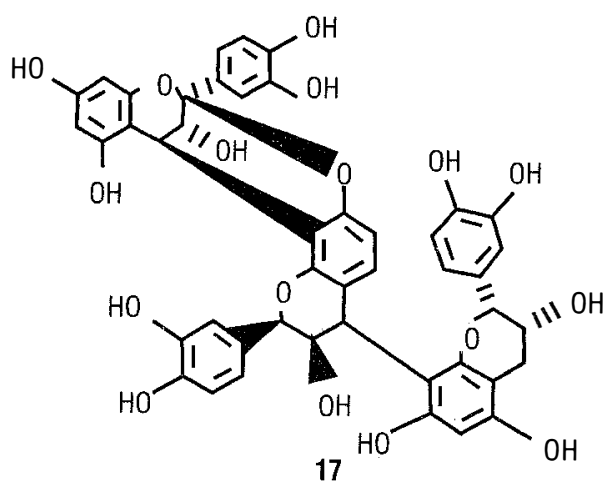

Fig 1. Metabolitos secundarios identificados en extractos de las plantas que inhibieron totalmente el efecto hemorrágico inducido en ratones por el veneno de Bothrops asper. 
metodología es sumamente sencilla y no requiere de equipo de laboratorio especializado. Se utilizó un procedimiento basado en la incubación de veneno y extractos previo a su inyección, con el fin de permitir la interacción de las toxinas con potenciales sustancias inhibidoras. Para fines de detectar compuestos antihemorrágicos esta metodología es mejor que los experimentos basados en inyección independiente de veneno y de los extractos (Gutiérrez et al. 1985), ya que en este caso la rapidez con que se desencadena el efecto en los tejidos hace difícil su inhibición por agentes neutralizantes. Por otra parte, se utilizó una alta razón extracto: veneno de 50:1 (peso por peso), con el fin de garantizar que no se perderían extractos que tuvieran muy bajas concentraciones de sustancias inhibitorias potencialmente importantes.

Al evaluarse 50 extractos, obtenidos de 48 especies de plantas seleccionadas con base en información etnobotánica, se observó que diez de ellos neutralizan totalmente esta actividad y con ocho adicionales se logra una inhibición parcial. Este alto grado de positividad en el tamizaje descrito abre posibilidades de purificar y caracterizar compuestos anti-hemorrágicos de las plantas estudiadas. Cuando se fraccionaron los extractos hidroalcohólicos mediante extracciones con solventes de polaridad creciente se observó que la mayor actividad inhibitoria se concentró en acetato de etilo y acuoso, con la excepción de Sapindus saponaria, cuya fracción diclorometánica presentó actividad inhibitoria. Al ser separadas cromatográficamente las fracciones que presentaron actividad anti-hemorrágica se aisló e identificó una serie de compuestos de naturaleza flavonoide, los cuales podrían ser responsables del efecto anti-hemorrágico observado.

Se ha descrito una importante serie de actividades farmacológicas de los flavonoides, tales como inhibición de la actividad plaquetaria y actividad antitrombótica en general (Chung et al. 1993). También presentan actividad antiinflamatoria y capacidad para inhibir fosfolipasas $\mathrm{A}_{2}$ (Gil et al. 1997). Se ha demostrado también que los flavonoides inhiben la oxidación de lipoproteínas de baja densidad (LDL), lo cual tiene importantes implicaciones en una potencial terapia de la aterosclerosis y de los trastornos cardiovasculares derivados de ella (Rankin et al. 1988). Terao et al. (1994) mostraron que catequinas como la epicatequina (compuesto 3) y galato de epicatequina (comuesto 7) son potentes agentes antioxidantes que previenen la peroxidación de fosfolípidos de membranas.

Son de interés, para las observaciones de este trabajo, hallazgos previos sobre la capacidad de los flavonoides de quelar iones metálicos. Por ejemplo, flavonoides como quercetina (compuesto 1), epicatequina (compuesto 3) y catequina (compuesto 4) han sido investigados por su acción quelante de hierro y cobre, lo cual podría tener aplicaciones en trastornos como hemocromatosis y enfermedad de Wilson (Dobbin y Hider 1990). La actividad hemorrágica de los venenos de serpiente es consecuencia de la acción enzimática de metaloproteinasas dependientes de zinc (Bjarnason y Fox 1994) y varias metaloproteinasas hemorrágicas han sido caracterizadas del veneno de B. asper (Borkow et al. 1993, Gutiérrez et al. 1995, Franceschi et al. 1999). Muchos inhibidores sintéticos de metaloproteinasas, tales como sales de EDTA y una serie de hidroxamatos, basan su mecanismo de acción en la quelación de átomo de zinc requerido para la catálisis (Borkow et al. 1997, Bottomley et al. 1998). Se ha descrito que catequinas aisladas del té negro son eficaces inhibidores de metaloproteinasas de matrix extracelular (Sazuka et al. 1997). Se sugiere que los flavonoides presentes en los extractos evaluados en el presente trabajo podrían inhibir la actividad hemorrágica de los venenos de serpientes mediante una acción quelante; esta hipótesis debe ser verificada en experimentos con flavonoides purificados.

Por otra parte, no se debe descartar la posible acción de los taninos condensados detectados en algunos de estos extractos, ya que estos compuestos, siendo formas poliméricas derivadas de la catequina y la epicatequina, 
podrían también interferir con el zinc ubicado en el sitio activo de las metaloproteinasas hemorrágicas. También debe considerarse la posibilidad de que las antocianinas, identificadas en algunos extractos, sean responsables de actividad anti-hemorrágica. Se ha descrito que estos compuestos disminuyen la fragilidad capilar y tienen acciones anti-inflamatorias (Harborne et al. 1988). Incluso las antocianinas han comenzado a sustituir a la rutina (compuesto 8), a la quercetina (compuesto 1) y a sus más potentes derivados sintéticos como la troxerutina (compuesto 11) y el pentabenzil éter de la quercetina (compuesto 12).

En conclusión, varios extractos de plantas costarricenses inhiben la actividad hemorrágica del veneno de $B$. asper en experimentos donde el veneno y los extractos se incuban previo a su inyección. La presencia en estos extractos de compuestos de naturaleza flavonoide, tales como catequinas, flavonas, antocianinas y taninos condensados, sugieren que este tipo de moléculas podrían tener una acción quelante del zinc requerido para la actividad enzimática de las metaloproteinasas hemorrágicas.

\section{AGRADECIMIENTOS}

Los autores agradecen al personal del Laboratorio de Productos Naturales del Departamento de Química de la Universidad Nacional (UNA) y del Instituto Clodomiro Picado, Universidad de Costa Rica, por su apoyo en las diferentes etapas de este trabajo. Asimismo, se agradece al Laboratorio de Productos Naturales de la Universidad de San Carlos, Sao Paulo, Brasil por las facilidades brindadas para la obtención de datos espectroscópicos de Resonancia Magnética de Protio y de Carbono 13. Esta investigación fue financiada por el Consejo Nacional de Investigaciones Científicas y Tecnológicas (CONICIT) de Costa Rica (proyecto 94 502-BID) y por el Instituto Nacional de Biodiversidad (INBio), con financiamiento de la Fundación MacArthur.

\section{RESUMEN}

Se evaluó la capacidad de extractos orgánicos de 48 especies de plantas costarricenses para neutralizar la actividad hemorrágica del veneno de la serpiente Bothrops asper (terciopelo). Los extractos se evaluaron mediante un bioensayo basado en inyecciones intradérmicas de veneno en ratones, o de mezclas veneno-extracto, seguidas de la cuantificación macroscópica de la hemorragia. Se observó una inhibición total de -la hemorragia con los extractos etanólico, de acetato de etilo y acuoso de Bursera simaruba, Clusia torresii, C. palmana, Croton draco, Persea americana, Phoebe brenesii, Pimenta dioica, Sapindus saponaria, Smilax cuculmeca y Virola koschnyi. El análisis químico de estos extractos permitió identificar catequinas, flavonas, antocianinas y taninos condensados, los cuales podrían jugar un papel en la inhibición del efecto hemorrágico debido a la capacidad de quelar el ion zinc requerido por las metaloproteinasas hemorrágicas para su acción.

\section{REFERENCIAS}

Aquino, R., M.L. Ciavatta \& F. De Simone. 1991. Catechins from Crøton draconoides. Fitoterapia 62: 454.

Bjarnason, J.B. \& J.W. Fox. 1994. Hemorrhagic metalloproteinases from snake venoms. Pharmac. Ther. 62 : 325-372.

Bolaños, R. 1982. Las serpientes venenosas de Centroamérica y el problema del ofidismo. Primera parte. Aspectos zoológicos, epidemiológicos y biomédicos. Rev. Cost. Ciencias Méd. 3: 165-184.

Borkow, G., J. M. Gutiérrez \& M. Ovadia. 1993. Isolation and characterization of synergistic hemorrhagins from the venom of the snake Bothrops asper. Toxicon 31: 1137-1150.

Borkow, G., J.M. Gutiérrez \& M. Ovadia. 1997. Inhibition of the hemorrhagic activity of Bothrops asper venom by a novel neutralizing mixture. Toxicon 35: 865-877.

Bottomley, K. M., W.H. Johnson \& D.S. Walter. 1998. Matrix metalloproteinase inhibitors in arthritis. J. Enzyme Inhibition 13: 79-101.

Campbell, J.A. \& W.W. Lamar. 1989. The Venomous Reptiles of Latin America. Cornell University, Nueva York. 425 p.

Céspedes, R., J. Chacón \& L. Mora. 1992. Estudio del contenido de extracto tánico de algunas plantas en Costa Rica. Ingeniería y Ciencia Química 14: 6-10. 
Chung, M.I., K.H. Gan, C.N. Lin, F. N. Ko \& C.M. Teng. 1993. Antiplatelet effects and vasorelaxing action of some constituents of Formosan plants. J. Nat. Prod. 56: 929-934.

Dobbin, P.S. \& R.C. Hider. 1990. Iron chelation therapy. Chem. Br. 26: 565-568.

Ferreira, L.A., O.B. Henriques, A.A.S. Andreoni, G.R.F. Vital, M.M.C. Campos, G.G. Habermehl \& V.L.G. de Moraes. 1992. Antivenom and biological effects of AR-turmerone isolated from Curcuma longa (Zingiberaceae). Toxicon 30: 1211-1218.

Franceschi, A., A. Rucavado, N. Mora \& J.M. Gutiérrez. 1999. Isolation and characterization of $\mathrm{BaH} 4$, a hemorrhagic metalloproteinase from the venom of the snake Bothrops asper. Toxicon (en prensa).

Gil, B., M.J. Sanz, M.C. Terencio, R. Gunasegaran, M. Payá \& M.J. Alcaraz. 1997. Morelloflavone, a novel biflavonoid inhibitor of human secretory phospholipase $A_{2}$ with antiinflammatory activity. Biochem. Pharmacol. 53: 733-740.

Gutiérrez, J.M. \& B. Lomonte. 1989. Local tissue damage induced by Bothrops snake venoms. Mem. Inst. Butantan 51: 211-223.

Gutiérrez, J.M. 1995. Clinical toxicology of snakebite in Central America, p. 645-665. In J. Meier \& J. White (eds.). Handbook of Clinical Toxicology of Animal Venoms and Poisons. CRC, Boca Raton, Florida.

Gutiérrez, J.M., G. León, G. Rojas, B. Lomonte, A. Rucavado \& F. Chaves. 1998. Neutralization of local tissue damage induced by Bothrops asper (terciopelo) snake venom. Toxicon 36: 1529-1538.

Gutiérrez, J.M., J.A. Gené, G. Rojas \& L. Cerdas. 1985. Neutralization of proteolytic and hemorrhagic activities of Costa Rican snake venoms by a polyvalent antivenom. Toxicon 23: 887-893.

Gutiérrez, J.M., M. Romero, C. Díaz, G. Borkow \& M. Ovadia. 1995. Isolation and characterization of a metalloproteinase with weak hemorrhagic activity from the venom of the snake Bothrops asper (terciopelo). Toxicon 33: 19-29.

Harborne, J.B. \& J.G. Renee. 1988. The anthocyanins, p. 1-20. In J.B. Harborne (ed.). The Flavonoids. Chapman and Hall, Londres.

Jacques, D. \& E. Haslam. 1974. Plant proanthocyanidins. Part II. Proanthocyanidin- $\mathrm{A}_{2}$ and its derivatives. J. Chem. Soc. Perkins 1: 2663-2671.
Kondo, H., S. Kondo, H. Ikezawa, R. Morita \& A. Ohsaka. 1960. Studies on the quantitative method for the determination of hemorrhagic activity of Habu snake venom. Jpn. J. Med. Sci. Biol. 13: 43-51.

Marham, K.R. \& B. Ternai. 1976. ${ }^{13}$ C-NMR of flavonoids. II. Flavonoids other than flavone and flavonol aglycones. Tetrahedron 32: 2607-2612.

Martz, W. 1992. Plants with a reputation against snakebite. Toxicon 30: 1131-1142.

Melo, P.A., M.C. do Nascimento, W.B. Mors \& G. SuárezKurtz. 1994. Inhibition of the myotoxic and hemorrhagic activities of Crotalid venoms by Eclipta prostrata (Asteraceae) extracts and constituents. Toxicon 32: 595-603.

Moreira, L., G. Borkow, M. Ovadia \& J.M. Gutiérrez. 1994. Pathological changes induced by $\mathrm{BaH} 1$, a hemorrhagic metallo roteinase isolated from Bothrops asper (terciopelo) snake venom, on mouse capillary blood vessels. Toxicon 32: 977-987.

Moreira, L., J.M. Gutiérrez, G. Borkow \& M. Ovadia. 1992. Ultrastructural alterations in mouse capillary blood vessels after experimental injection of venom from the snake Bothrops asper (terciopelo). Exp. Mol. Pathol. 57: 124-133.

Rankin, S.M., J.R.S. Hoult \& D.S. Leake. 1988. Effects of flavonoids on the oxidative modification of low density lipoproteins by macrophages. Br. J. Pharmacol. 95: 727.

Rosenfeld, G. 1971. Symptomatology, pathology and treatment of snake bites in South America, p. 345-384. In W. Bucherl \& E. Buckley (eds.). Venomous Animals and Their Venoms. Academic Press, Nueva York.

Sazuka, M., H. Imazawa, Y. Shoji, T. Mita, Y. Hara \& M. Isemura. 1997. Inhibition of collagenases from mouse lung carcinoma cells by green tea catechins and black tea theaflavins. Biosci. Biotech. Biochem. 61, 1504-1506.

Terao, J., M. Piskula \& Q. Yao. 1994. Protective effects of epicatechin gallate and quercetin on lipid peroxidation in phospholipid bilayer. Arch. Biochem. Biophys. 308: 278-284.

Warrell, D.A. 1996. Clinical features of envenoming from snake bites, p. 64-76. In C. Bon \& M. Goyffon (eds.) Envenomings and Their Treatments. Fondation Marcel Mérieux, Lyon.

Woessner, J.F. 1994. The family of matrix metalloproteinases. Ann. N.Y. Acad. Sci. 732: 11-21. 